\title{
Application of Concentrating Luminescent Down-shifting Structures to CdS/CdTe Solar Cells with Poor Short Wavelength Response
}

\author{
Thomas S. Parel ${ }^{\mathrm{a}, *}$, Lefteris Danos ${ }^{\mathrm{b}}$ and Tomas Markvart ${ }^{\mathrm{a}}$ \\ ${ }^{a}$ Solar Energy Laboratory, University of Southampton, Southampton SO17 1BJ, United Kingdom \\ ${ }^{\mathrm{b}}$ Department of Chemistry, Lancaster University, Lancaster LA1 4YB, United Kingdom \\ * Corresponding author. Tel.: +44 7514807 459. Email address: T.S.Parel@southampton.ac.uk
}

\begin{abstract}
A method to exploit both the wavelength shifting and light concentrating properties of luminescent plates in order to increase the current output of $\mathrm{CdS} / \mathrm{CdTe}$ solar cells has been investigated. The application of concentrating luminescent down-shifting structures onto CdS/CdTe solar cells in order to combine the benefits of light concentration and wavelength shifting properties of luminescent plates is proposed. This technique is particularly effective in $\mathrm{CdS} / \mathrm{CdTe}$ solar cells with a poor short wavelength response. An increase in the current output of close to $10 \%$ was measured on application of concentrating luminescent down-shifting structures compared to near $5 \%$ without light concentration to such solar cells. Concentrating luminescent down-shifting structures containing multiple fluorescent species allowed the absorption ability of the device to be extended to a wider range of wavelengths. This combined with strong energy transfer that red-shifts photons to wavelengths with even better solar cell response is seen to result in increases greater than $20 \%$ in the short circuit current output of $\mathrm{CdS} / \mathrm{CdTe}$ solar cells. A general analytical expression that models different methods of applying of luminescent plates to solar cells is proposed which describes the performance of concentrating luminescent down-shifting structures well and can be used to separate the contributions of luminescence and incident excitation photons on the current output. These expressions can also be used to estimate the transport and reflection losses in these devices.
\end{abstract}

Keywords - energy, energy conversion, photovoltaic systems, solar energy, solar power generation.

\author{
Abbreviations \\ C-LDS: concentrating luminescent down-shifting structures \\ LDS: luminescent down-shifting structures \\ LP: luminescent plate \\ LSC: luminescent solar concentrator \\ O: BASF Lumogen F series Orange240 dye \\ V: BASF Lumogen F series Violet 570 dye \\ Y: BASF Lumogen F series Yellow083 dye
}




\section{Introduction}

The main barrier to the wide spread utilization of solar energy for electricity generation is higher costs as compared to conventional energy sources. Different strategies have been investigated to reduce the cost of solar electricity [1]. Examples include research into the cost reduction of high efficiency solar cells and concentration of solar energy [2][3].

One strategy to increase the efficiency of some solar cells is to improve their response to short wavelength photons. In certain solar cells such as those based on cadmium telluride ( $\mathrm{CdTe})$, for example, the buffer/window layer consists of cadmium sulfide (CdS) that absorbs short wavelength light [4][5].

Photon management, through wavelength shifting or light concentration, can be achieved using luminescent plates (LP). These devices have the potential to improve the short wavelength conversion efficiency of photovoltaic systems and reduce costs by concentrating light onto smaller solar cells [6]. LPs consist of molecules that absorb solar photons and re-emit light at a longer wavelength. Non-concentrating LPs, commonly referred to as luminescent down-shifting structures (LDS), have been used to shift the wavelength of solar photons to regions where the performance of the solar cell is superior [7] (see Fig. 1 (b)).

This has been undertaken for a number of different solar cells including crystalline silicon (cSi) [8][9], amorphous silicon [7], CdS/copper sulfide [7], CdS/CdTe [10][11][12] and copper indium gallium selenide (CIGS) [13][14], an extensive list including efficiency improvements can be found in [15].

Direct comparisons on the effect of different LDS in different studies are, however, difficult since the enhancements observed strongly depend on the external quantum efficiency (EQE) of the solar cell (as well as luminescent dye combinations used). For example, the application of a LDS containing BASF Lumogen F series dyes on CdS/CdTe resulted in widely differing enhancement estimates in [10] compared to ray tracing results in [16] due to the large difference in the response of the different CdS/CdTe solar cells considered. The performance of different types of LPs are affected by common effects like photon recycling via re-absorption of emitted fluorescence [17][18], reflection losses, light trapping structures, position of the LP etc. The effect of reflection [19], light trapping structures [19], LP configuration [20][21] as well as radiative and non-radiative recombination [20][21] in devices known as down-converters have previously been investigated. Down-converters are similar to LDS but convert a high energy photon into multiple lower energy photons.

LPs can concentrate light by trapping a significant part of the luminescence within the device through total internal reflection [22] i.e. Fig. 1 (a). These primarily concentrating devices will be referred to as luminescent solar concentrators (LSC) and are distinguished from LDS in that light concentration rather than spectral matching is the primary goal in these devices.

Though LSCs, as shown in Fig. 1(a) are normally edge mounted, bottom mounted configurations similar to LDS have also been proposed [23] and have been investigated for increasing the performance of cSi solar cells [24][25][26][27]. In [28] a bottom mounted LSC was considered when calculating the efficiency limit of LSCs coupled to solar cells of different band gaps. The 
advantage of such a configuration is that it ensures that concentration onto the solar cell acts to augment the performance of directly excited solar cells. It has also been suggested that these devices could be suitable for building integrated systems and recent calculations indicate a reduction in the cost/watt of such systems coupled to cSi to be around 28\% [27].

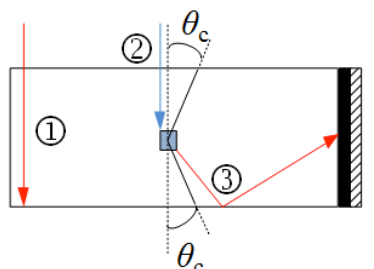

(a)

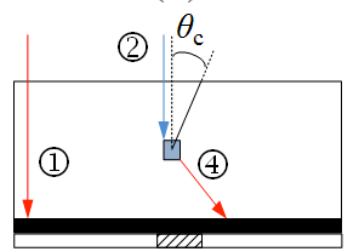

(b)

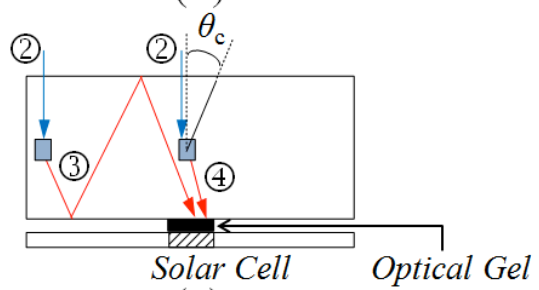

(c)

Fig. 1. Comparison of different LSC geometries (the blue rectangle represents a luminescent molecule): (a) edge mounted LSC,

(b) LDS and (c) C-LDS. The different rays shown are 1) long wavelength incident ray not absorbed by LP, 2) short wavelength incident ray absorbed by LP, 3) ) trapped luminescence reaching the solar cell and 4) luminescence directly reaching solar cell/solar cell substrate.

The concept of LDS and bottom mounted LSCs is extended one step further and the application of concentrating luminescent down-shifting structures (C-LDS) that simultaneously improve the short wavelength response of CdS/CdTe solar cells and also concentrate light is proposed. A schematic is shown in Fig. 1 (c). This technique is particularly effective for improving the performance of $\mathrm{CdS} / \mathrm{CdTe}$ solar cells with a poor short wavelength response. Since a C-LDS is simply applied on top of the solar cell, it's a simple and cost effective method of increasing performance without introducing additional processing to the solar cell. To highlight the effect of the application of C-LDS, CdS/CdTe solar cells with a relatively thick CdS layer and therefore a poor short wavelength spectral response have been investigated for application of C-LDS. For higher efficiency solar cells lower improvements in performance from the wavelength shifting aspect of C-LDS will be expected though increases in performance due to light concentration is likely to be maintained or be even larger depending on the EQE of the solar cell.

A C-LDS has a number of key advantages. C-LDS can be used to collect light from regions where, for example, shading due 
to front contacts or gaps between modules prevents capture of solar energy. Additionally, the poor short wavelength response found in many solar cells is simultaneously improved. This offers the possibility of achieving higher efficiencies on existing solar cell devices.

Furthermore, in central and northern Europe around 50\% of the solar energy reaching the ground is diffuse [29]. Since LPs absorb light from all direction, C-LDS can concentrate diffuse light unlike geometric concentrators. This also means that there is no need for the solar tracking required in systems using geometric concentrators. This avoids the added costs associated with the mechanical systems required for solar tracking and also reduces maintenance and benefits from the reliability of systems lacking moving components. The high content of blue light in diffuse radiation enhances the effect of wavelength shifting since these devices are able shift the short wavelength diffuse light to longer wavelengths that are utilized by the solar cell with a higher efficiency. C-LDS therefore benefit from advantages of both LSCs and LDS.

C-LDS were first proposed and experimentally investigated for application to cSi solar cell in [8]. The concept of combining spectral matching with solar concentration for cSi solar cells was further developed in [30]. In this publication a theoretical model was developed to describe concentration of light (using back scattering) onto a bottom mounted solar cell and the results were compared to experiments. The addition of fluorescent molecules to the device to incorporate spectral matching was investigated experimentally but the model was not extended to include this additional complexity.

More recently in [31] the technique of combining spectral matching and light concentration was used to concentrate as well as shift photons to the low reflectivity region of a silicon microcell array. Simultaneous use of light concentration and spectral matching has also been shown to increase the current output of a photo-electro-chemical cell attached to one edge of a liquid C-LDS [32]. However, to our knowledge the application of a C-LDS to CdS/CdTe has not been investigated previously.

A number of studies have, however, investigated LDS incorporated into module encapsulation layers slightly larger than the solar cell active area. This has been undertaken for different solar cells such as cSi [9], CIGS [13] [14] and CdS/CdTe [33]. Also, in [10] for example, a LDS applied on a slightly smaller CdS/CdTe solar cell was investigated. However, even though there might be some concentration of light due to the differences in sizes between the solar cell and the LDS in these devices, these studies were focused on the wavelength shifting property of LPs.

The results of experimental and theoretical analysis of these devices will be presented. It also appears that the use of energy transfer that occurs most likely through re-absorption [23] but possibly also through Förster resonance energy transfer (FRET) ([34] or see for example [35]) is able to further improve the performance of these systems.

\section{Theory}

The excitation photon flux is assumed to consist of uniform, parallel rays incident normal to the top surface of the LP. A 
solar cell is attached to this device at an inclination angle, $\phi$, with respect to the top surface of the LP i.e. for conventional edge mounted LSCs this angle is equal to $90^{\circ}$ while for bottom mounted LSCs, LDS and C-LDS this angle is equal to $0^{\circ}$. The short circuit current output, $I_{\mathrm{sc}}$, of the solar cell due to incident solar photons of a particular excitation wavelength, $\lambda$, is given by:

$$
\begin{aligned}
I_{\mathrm{c}}(\lambda) & =I_{\mathrm{f}}(\lambda)+I_{\mathrm{d}}(\lambda) \\
& =q\left\{1-R_{\text {top }}(\lambda)\right\} Q_{\mathrm{c}}(\lambda) Q_{\mathrm{a}}(\lambda) E Q E_{\mathrm{f}}(\lambda) \dot{N}_{\text {inc }}(\lambda)+\frac{q\left\{1-R_{\text {top }}(\lambda)\right\} A_{\text {cell }} \cos \phi}{A_{\text {col }}}\left\{1-Q_{\mathrm{a}}(\lambda)\right\} E Q E(\lambda) \dot{N}_{\text {inc }}(\lambda)
\end{aligned}
$$

where $I_{\mathrm{f}}$ is the current produced in the solar cell due to luminescence reaching the solar cell, $I_{\mathrm{d}}$ is the current produced in the solar cell due to the direct incidence of excitation light, $q$ is the charge of an electron, $R_{\text {top }}$ is the reflectance from the top surface of the LP, $Q_{\mathrm{c}}$ is the collection efficiency defined as the fraction of photons of a given excitation wavelength absorbed by the LP that results in a luminescent photon reaching the solar cell, $Q_{\mathrm{a}}$ is the absorption efficiency i.e. fraction of photons of a given excitation wavelength absorbed by the device, $E Q E_{\mathrm{f}}$ is the average external quantum efficiency (EQE) of the solar cell due to the luminescence incident on it, EQE is the EQE of the solar cell, $\dot{N}_{\text {inc }}$ is the photon flux incident on the top surface of the LP, $A_{\text {cell }}$ is the top surface area of the solar cell and $A_{\text {col }}$ is the top surface area of the LP. This is a more general expression than what is given in [36] and describes the current output of solar cells coupled to LSCs, LDS or C-LDS.

The variation of $Q_{\mathrm{c}}$ and $E Q E_{\mathrm{f}}$ with the excitation wavelength can be explained as follows. Re-absoprtion occurs when the emitted luminescence is absorbed due to a partial overlap between the absorption and emission bands of the luminescent species. Though luminescence emitted by a molecule is independent of the excitation wavelength, the luminescence reaching a solar cell travels a finite distance leading to a certain amount of re-absorption, which distorts the luminescence spectrum. The probability of re-absorption depends upon the path length travelled which in turn depends upon the depth at which the excitation photon is absorbed. The probability of the latter is related to the absorption coefficient of the LP and varies with the excitation wavelength. Therefore the spectral distribution of the luminescence that reaches the solar cell can vary with the excitation wavelength. This explains why $E Q E_{\mathrm{f}}$ might also change at different excitation wavelengths.

It can be shown that $Q_{\mathrm{c}}$ depends upon the re-absorption probabiltiy [17][18]. The variation in the re-absorption probability with the excitation wavelength also makes $Q_{\mathrm{c}}$ dependent on the excitation wavelength. Furthermore, the luminescence collected from a LP containing a number of different luminescent species will be determined by additional factors such as the energy transfer efficiency between the different luminescent molecules present and the absorption coefficient of each luminescent species at a given excitation wavelength.

$Q_{\mathrm{c}}$ and $E Q E_{\mathrm{f}}$ is, therefore, only independent of the excitation wavelength in specific systems where, for example, the 
absorbance does not vary significantly with the excitation wavelength and there is, either, only one type of luminescent molecule or there exists perfect energy transfer to the acceptor molecules in the case there are different luminescent species.

In such a system if $R_{\text {top }}$ is also assumed to be independent of the excitation wavelength, the current output of a solar cell attached to the LP reduces to:

$$
I_{\mathrm{c}}(\lambda)=C Q_{\mathrm{a}}(\lambda) \dot{N}_{\text {inc }}(\lambda)+D\left\{1-Q_{\mathrm{a}}(\lambda)\right\} \dot{N}_{\text {inc }}(\lambda) E Q E(\lambda),(2)
$$

where $C$ and $D$ are constants. From Equation 2 it is possible to obtain the contribution of wavelength shifting and light concentration on the current output of the solar cell and also to estimate the current output at excitation wavelengths not measured.

The constant $C$ can be obtained by fitting the experimentally measured current output in wavelength regions where the solar cell has a low EQE. The constant $D$ can similarly be obtained by fitting the measured current output in regions where the absorbance of the LSC is close to zero.

From the constant $D, R_{\text {top }}$ can be obtained and then from $C, Q_{\mathrm{c}}$ can also be obtained if $E Q E_{\mathrm{f}}$ can be approximated to be equal to a weighted average of luminescence exiting the LP surface coupled to a solar cell. These parameters can be used to quantify losses. By comparing the $Q_{\mathrm{c}}$ for different fabrication methods or configurations i.e. for example, with and without edge mirrros, losses can be identified and the design of the LP adapted accordingly.

\section{Materials and methods}

The LPs used in this study consist of thin film spin coated layers containing fluorescent dyes embedded within poly(methyl methacrylate) (PMMA, Microchem 950 C 10) of refractive index 1.49 (at $587.6 \mathrm{~nm}$ ). BASF, Lumogen F series dyes Violet570 (V), Yellow083 (Y) and Orange240 (O) are the fluorescent species used.

The dye-PMMA solutions were put in a sonicator for 30 minutes and left overnight in order to ensure complete dissolution. The resulting solutions were deposited on the surface of a $20 \times 20 \mathrm{~mm}$ optical glass (BK7 windows) substrate by spin coating (Laurell) at $500 \mathrm{rpm}$ resulting in a thin film thickness of $8 \mu \mathrm{m}$. The glass substrates have a refractive index of 1.52 and reflection loss of $8.1 \%$ for 2 surfaces (at $587.6 \mathrm{~nm}$ ). A glass thickness of $1 \mathrm{~mm}$ was selected. Due to the similar refractive index of the glass and the PMMA, the LP can be treated as a homogenous plate with an effective absorption coefficient as detailed in [18].

The following naming convention has been adopted for different samples: the dye concentration in the PMMA solution, i.e. before deposition, is written in units of $\mathrm{g} / \mathrm{l}$ after the letter representing each dye. The peak absorbance of the sample is also written in square brackets. For example a sample consisting of $4 \mathrm{~g} / \mathrm{l} \mathrm{Y}$ dye and $2 \mathrm{~g} / \mathrm{l} \mathrm{O}$ dye with a peak absorbance of 1.8 will be denoted by $\mathrm{Y} 4, \mathrm{O} 2[1.8]$.

The absorbance (denoted by $A$ ) of the LPs were measured using a Bentham spectrometer and an IL1 100 W Halogen light 
source, and was linked to $Q_{\mathrm{a}}$ using the following expression:

$$
Q_{\mathrm{a}}(\lambda)=1-10^{-A(\lambda)}
$$

The top and bottom fluorescence emitted from the LPs was detected using an Avantes (AvaSpec-2048) spectrometer and an 89 North PhotoFluor II $200 \mathrm{~W}$ Metal Halide lamp (excitation at $440 \mathrm{~nm}$ ). The setup for measuring the top fluorescence is shown in Fig. 2. The first generation fluorescence i.e. the re-absorbance free fluorescence was obtained by measuring the top fluorescence of low dye concentration LPs.

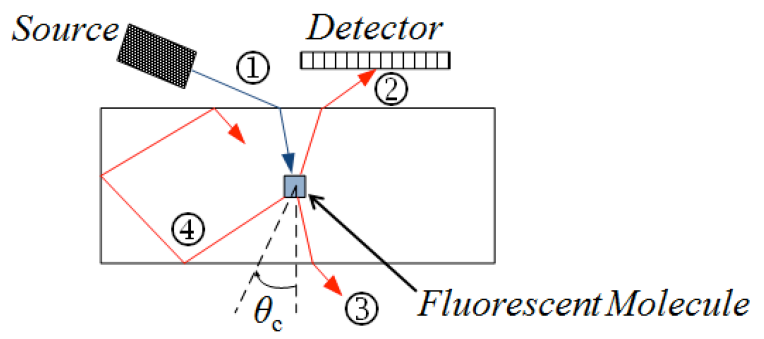

Fig. 2. Setup for the measurement of emission from the top surface of a LP. The different rays shown are 1) excitation ray from the source, 2) escape cone luminescence lost from the top surface 3) escape cone luminescence lost from the bottom surface and 4) trapped luminescence.

A solar simulator (T.S. Space Systems) equipped with a $300 \mathrm{~W}$ Xenon lamp approximating the AM1.5 spectrum and a filter selection wheel (350-950 nm, step $50 \mathrm{~nm}$ ) was used to measure the current output of the solar cell. The Xenon lamp was calibrated against a silicon standard solar cell calibrated at the National Renewable Energy Laboratory (NREL) for standard test conditions (AM $1.5,1000 \mathrm{~W} / \mathrm{m}^{2}$ at $\left.25^{\circ} \mathrm{C}\right)$.

The LPs were applied on top of CdS/CdTe solar cells fabricated at the Centre for Solar Energy Research (CSER), Glyndŵr University (see, for example [37] for the methodology used to manufacture them). These solar cells have been fabricated specifically for wavelength shifting applications and contain a relatively thick CdS buffer layer (380 $\mathrm{nm})$. The solar cell performance is shown in Figure 3. In operation, a fixed surface area $(5 \times 5 \mathrm{~mm})$ of the solar cell was continuously exposed to incoming light.

Index matching gel (Thorlabs) was used to couple the LP to the solar cells. In the C-LDS configuration, the LP must be much larger than the solar cell and selective index matching to areas only on top of the solar cell should allow a significant amount of light to be concentrated onto the solar cell (see Fig. 1 (c)) and also improve the short wavelength spectral response of the cell. A uniform application of index matching gel extending to the solar cell substrate, on the other hand, should not produce any 
concentration effect and is, therefore, expected to give a lower current output. To highlight the improvements, the results of current outputs measured for these two different methods of applying index matching gel at the solar cell-LP interface will be presented.

Current was also measured for an un-doped LP applied on the CdS/CdTe solar cell. This was used to compare the effect of wavelength shifting and light concentration while ensuring that effects associated with the substrate and PMMA are not taken into consideration.

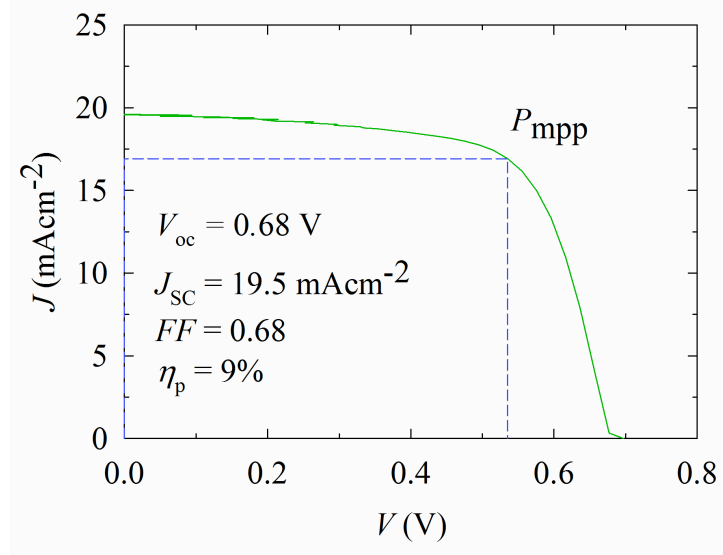

Fig. 3. CdS/CdTe solar cell performance.

\section{Results and discussion}

This section details experimental results of the current output of CdS/CdTe solar cells with LDS and C-LDS applied on top. An attempt has also been made to estimate the current contribution of luminescent light based on the absorption efficiency, incident photon flux and the EQE of the solar cell.

Figure 4 shows the absorbance and emission (i.e. first fluorescence) spectra of the $\mathrm{Y}$ and $\mathrm{O}$ dye, the EQE of the CdS/CdTe solar cell used and the photon flux distribution of a typical Xenon lamp. It is readily apparent that absorbance by the $\mathrm{O}$ and $\mathrm{Y}$ dyes takes place in regions where the EQE of this solar cell is poor. The fluorescence of the $\mathrm{O}$ dye occurs however at wavelengths converted with a much higher EQE.

The large overlap between the emission of the $\mathrm{Y}$ dye and the absorbance of the $\mathrm{O}$ dye indicates the possibility of energy transfer that should result in a red shift of the emission in samples containing a mixture of both dyes. The benefit of this is that emission of fluorescence at longer wavelengths results in better electrical conversion by the solar cell.

Measurements under white light (Xenon lamp) of C-LDS with different Y dye concentrations applied on CdS/CdTe solar cells were conducted so as to get a range of suitable dye concentrations. The results indicate that a $\mathrm{Y}$ dye concentration of $5 \mathrm{~g} / \mathrm{l}$ gave the greatest increase in solar cell current output as shown in Figure 5. After this concentration a reduction is observed possibly due to diminishing returns in $Q_{\mathrm{a}}$ and increases in re-absorption losses. 


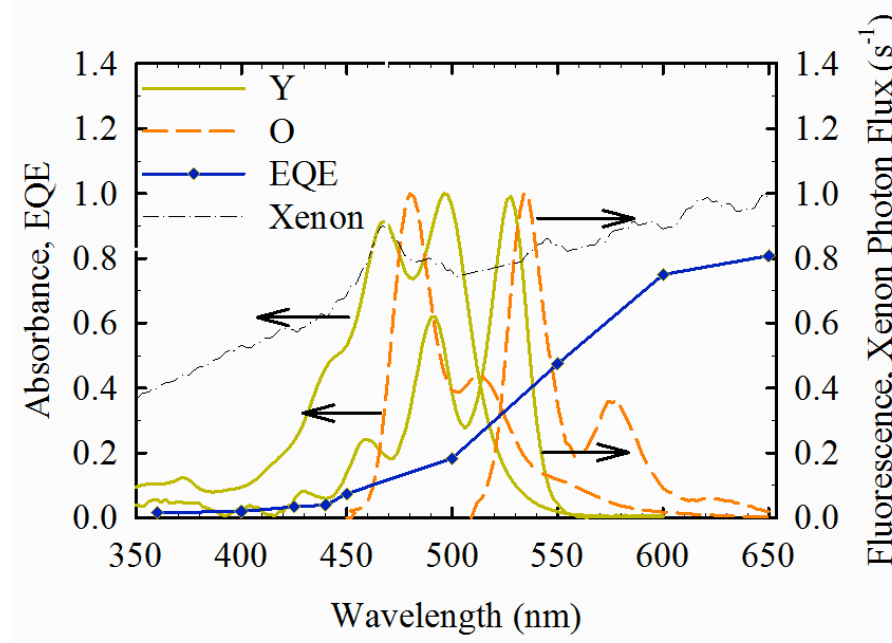

Fig. 4. Absorbance and first generation fluorescence spectrum of $\mathrm{Y}$ and $\mathrm{O}$ dyes. The EQE of the CdS/CdTe solar cell used is also plotted. The peaks have been normalized to unity. The absolute value of the Xenon lamp photon flux at $650 \mathrm{~nm}$ is $2.7 \mathrm{x} 10^{18}$ $\mathrm{s}^{-1} \mathrm{~m}^{-2} \mathrm{~nm}^{-1}$

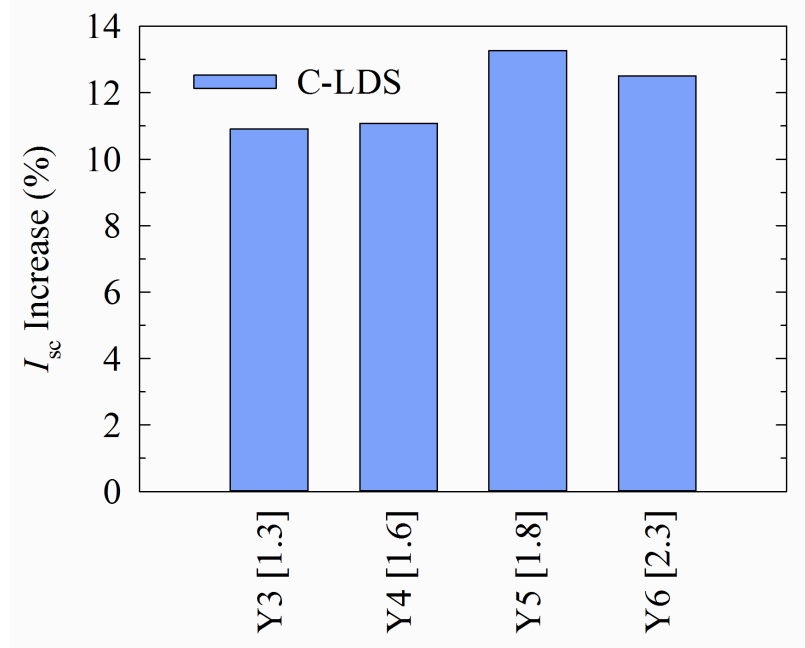

Fig. 5. Measured increase in $I_{\mathrm{sc}}$ relative to un-doped PMMA LDS under white Xenon light for Y dye C-LDS.

Subsequently, LPs of different mixtures of V, Y and O dye were fabricated and tested as LDS and C-LDS. Figure 6 shows the relative increase in $I_{\mathrm{sc}}$ on application of these LPs on top of CdS/CdTe solar cells (measurements were again conducted under calibrated Xenon white light). The results indicate that $\mathrm{Y}$ and $\mathrm{O}$ dyes are superior for light concentration most likely because there are more excitation photons at the absorbing wavelengths of these dyes. V dye is seen to be useful primarily for enhancing the wavelength shifting effect since the EQE of the solar cell is very poor at the absorbing wavelengths of this dye. A total dye concentration beyond $6 \mathrm{~g} / \mathrm{l}$ was not observed to increase the current output of C-LDS possibly due to greater re-absorption. Indeed, in some samples higher dye concentrations are seen to lead to a smaller enhancement in current output. From the results 
it appears that, among the samples studied, the Y4,O2 [1.8] C-LDS with a total dye concentration of $6 \mathrm{~g} / 1$ gives the largest increases in current output.

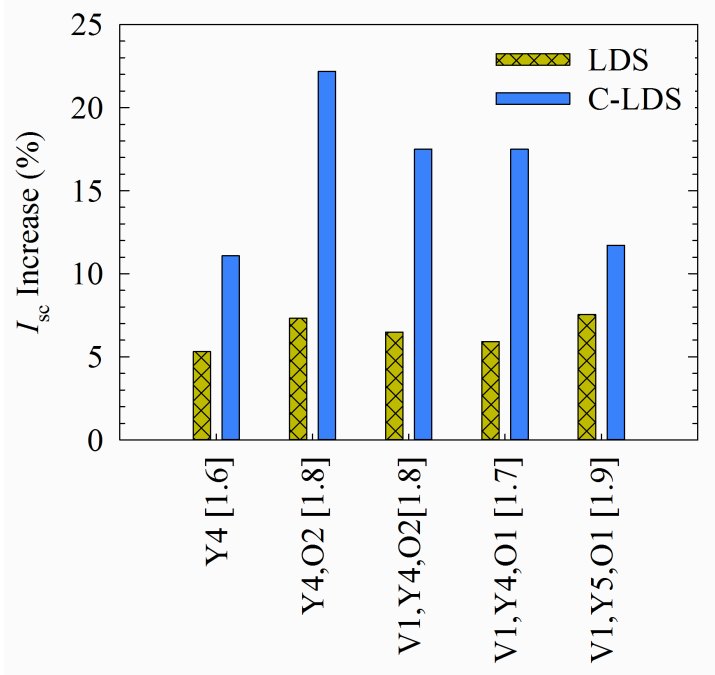

Fig. 6. Measured increase in $I_{\mathrm{sc}}$ relative to un-doped PMMA LDS under white Xenon light for multi-dye LPs.

It is seen that for a Y4 [1.6] LDS, a close to 5\% increase in current output was measured while for the same LP as a C-LDS a resulting current increase near $10 \%$ is observed due to concentration of photons. This is further improved to over $20 \%$ on application of the Y4,O2 [1.8] C-LDS.

The current output for excitation with photons of different wavelengths has also been measured and Equation 2 has been used to calculate a theoretical fit for different samples as shown in Figure 7. In this manner the constants $C$ and $D$ were also obtained. For the theoretical fit, the photon flux for a typical Xenon lamp (shown in Fig. 4) has been used as the incident photon flux. The fit is seen to match the current measurements rather well in spite of the large variation in the absorbance of these LPs (Figure 9). This indicates that the assumption of constant $Q_{\mathrm{c}}$ and $E Q E_{\mathrm{f}}$ with the excitation wavelength is a good approximation in the devices fabricated. 


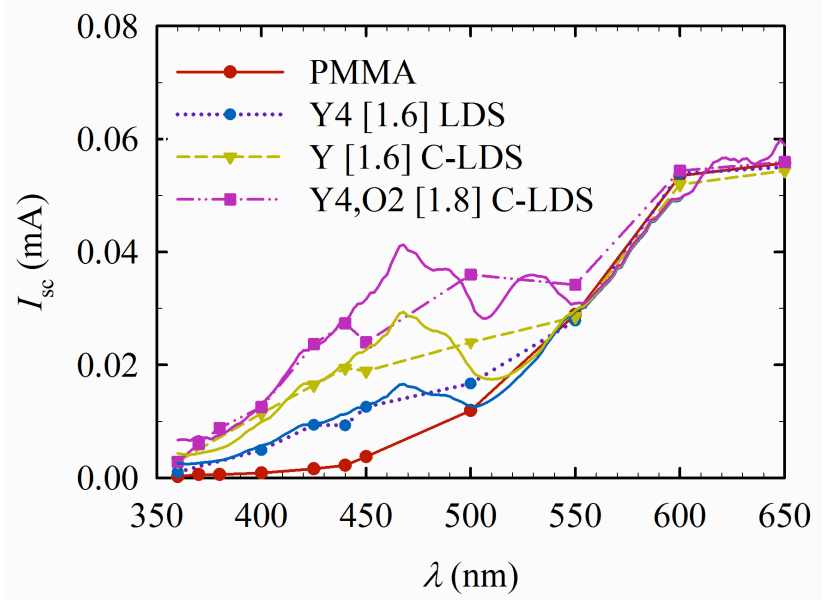

Fig. 7. Enhancement of current output of solar cells coupled to LDS and C-LDS, light concentration and the use of multiple dyes with energy transfer. Fit based on Equation 2 also shown as solid lines.

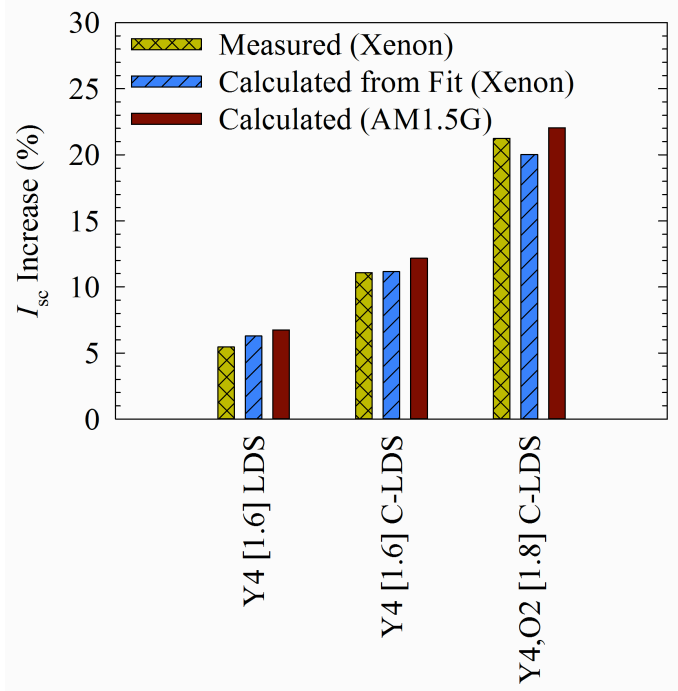

Fig. 8. Measured and calculated (from fit based on Equation 2) increase in $I_{\mathrm{sc}}$ relative to un-doped PMMA LDS under white Xenon light. 


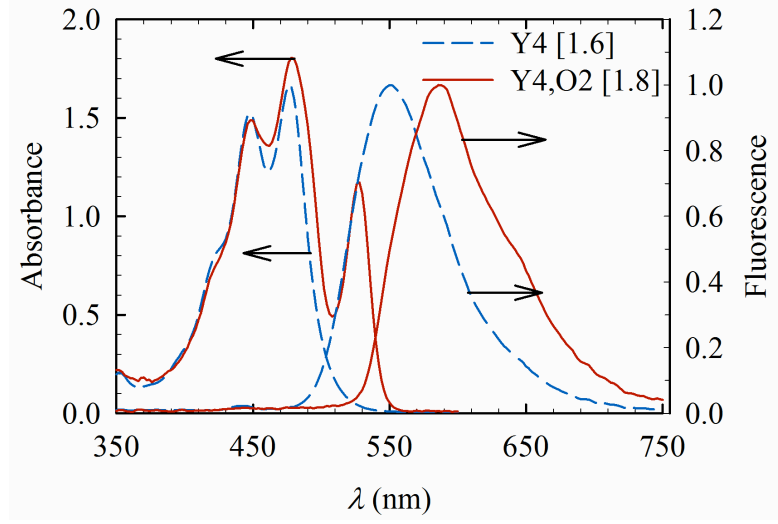

Fig. 9. Absorbance and bottom fluorescence of the Y4 [1.6] and Y4,O2 [1.8] LPs.

By integrating the theoretical fits based on Equation 2, a calculated increase in $I_{\mathrm{sc}}$ has been obtained and is compared to the experimentally measured value as shown in Figure 8. A reasonably good correlation is observed. Figure 8 also shows the increase in $I_{\mathrm{sc}}$ predicted under AM1.5G that has been calculated by using the AM1.5G spectrum for $N_{\text {inc }}^{\&}$ in Equation 2.

Figure 9 shows the absorbance and fluorescence escaping the bottom surface of the Y4 [1.6] and Y4,O2 [1.8] LPs (the fluorescence was subject to multiple re-absorption and re-emission events). It is clear that for incident photons with wavelengths below $450 \mathrm{~nm}$ the absorbance is not significantly different for the two different LPs. The bottom fluorescence (excitation at 440 $\mathrm{nm}$ ) is, however, seen to be red-shifted for the $\mathrm{Y} 4, \mathrm{O} 2[1.8] \mathrm{LP}$ as compared to the Y4 [1.6] LP. This indicates energy transfer from the $\mathrm{Y}$ to the $\mathrm{O}$ dye with emission taking place mostly in the $\mathrm{O}$ dye. The larger $\mathrm{EQE}$ of the solar cell corresponding to the wavelength distribution of the bottom fluorescence of the Y4,O2 [1.8] LP explains the larger current output of the solar cell coupled to this as a C-LDS as compared to coupling to the Y4 [1.6] LP as a C-LDS as shown Figure 7, even at excitation wavelengths with a similar absorbance.

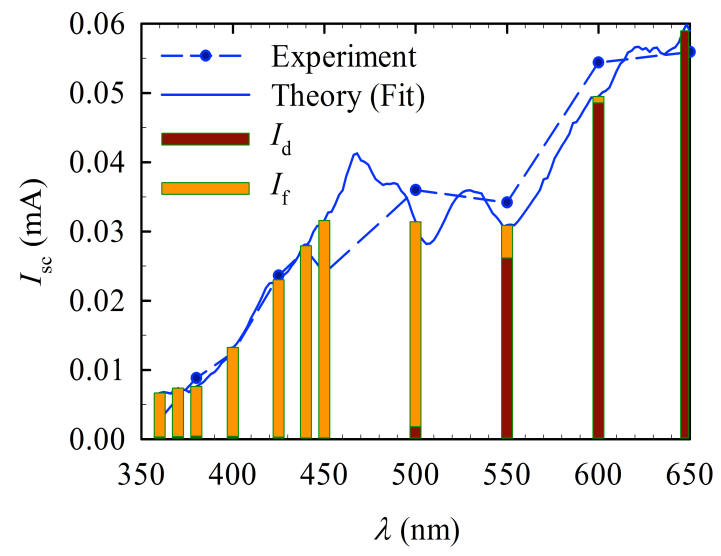

Fig. 10. Contribution of Y4,O2 [1.8] C-LDS and excitation light on the current output of the solar cell: obtained from a fit based on Equation 2. 
The contribution of fluorescence, $I_{\mathrm{f}}$, and direct excitation light, $I_{\mathrm{d}}$, on the current output have also been determined from (Equation 2) and is plotted in Figure 10 for the Y4,O2 [1.8] C-LDS. As shown clearly in this figure Equation 2 allows an estimation of the effect of the LP even at wavelengths where current is due to a mixture of direct and luminescent photons. It is clear from Figure 10 that current output due to short wavelength excitation photons is primarily due to the effect of the LP, however as the EQE of the solar cell increases and the absorbance of the LP decreases, the current output is due to a mixture of luminescent and direct excitation photons. Finally, at long wavelengths where the LP does not absorb light, the current output is entirely due to the direct electrical conversion of excitation photons. The constants $C$ and $D$ obtained from Equation 2 can also be used to estimate the reflectance and transport losses in LPs which will be useful for optimizing the design of such systems.

\section{Conclusions}

The application of C-LDS to CdS/CdTe solar cells with a poor short wavelength response has been proposed and was investigated experimentally and using a theoretical expression. C-LDS is superior to conventional edge mounted LSCs in that both direct light and luminescence is utilized. It is also more effective than a non-concentrating LDS system in increasing the current output of solar cells since these structures can simultaneously improve the short wavelength response of solar cells and concentrate light. The increase in current output measured for a CdS/CdTe solar cell coupled to a C-LDS is seen to be over $10 \%$ larger than with an un-doped LP. This is significantly greater than the 5\% increase measured on applying the same LP as a LDS. It has been shown that the use of multiple fluorescent species with energy transfer further enhances the effect of C-LDS, improving the current output of coupled CdS/CdTe solar cells by over $20 \%$. The contribution of LPs to the current output of the attached $\mathrm{CdS} / \mathrm{CdTe}$ solar cell has also been determined from a general theory that describes different methods of coupling LPs to solar cells. It is also shown that the expressions used are in good agreement with experimental measurements.

\section{Acknowledgements}

This work has benefitted from the Sustainable Power Generation and Supply, Photovoltaics for the 21st Century (SuperGen PV21) Initiative and the Engineering and Physical Sciences Research Council (EPSRC).

\section{References}

[1] A. Luque, S. Hegedus, Status, trends, challenges and the bright future of solar electricity from photovoltaics, in: A. Luque, S. Hegedus, Handbook of Photovoltaic Science and Engineering, Wiley, Chichester, 2003, pp. 1-43.

[2] N.S. Lewis, Towards cost effective solar energy use, Science 315 (2007) 798-801. 
[3] D.J. Friedman, R.R. King, R.M. Swanson, J. McJannet, D. Gwinner, Towards 100 gigawatts of concentrator photovoltaics, IEEE Journal of Photovoltaics 3 (2013) 1460-1463.

[4] B.S. Richards, K.R. McIntosh, Overcoming the poor short wavelength response of CdS/CdTe photovoltaic modules via luminescence down-shifting: ray tracing simulations, Progress in Photovoltaics: Research and Applications 15 (2006) 27-34.

[5] D.E. Swanson, R.M. Geisthardt, J.T. McGoffin, J.D. Williams, J.R. Sites, Improved CdTe solar-cell performance by plasma cleaning the TCO layer, IEEE Journal of Photovoltaics 3 (2013) 838-842.

[6] M.G. Debjie, P.P.C. Verbunt, Thirty years of luminescent solar concentrator research: solar energy for the built environment, Advanced Energy Materials 2 (2012) 12-35.

[7] H.J. Hovel, R.T. Hodgson, J.M.Woodall. The effect of fluorescent wavelength shifting on solar cell spectral response, Solar Energy Materials 2 (1979) 19-29.

[8] D.Sarti, F. Poull, P. Gravisse, Transformation du rayonnement solaire par fluorescence: application a l'encapsulation des cellules, Solar Cells 4 (1981) 25-35.

[9] K.R. McIntosh, G. Lau, J.N. Cotsell, K. Hanton, D.L. Bätzner, F. Bettiol, B.S. Richards, Increase in external quantum efficiency of encapsulated silicon solar cells from a luminescent donw-shifting layer, Progress in Photovoltaics: Research and Applications 17 (2009) 191-197.

[10] T. Maruyama, R. Kitamura, Transformation of the wave length of the light incident upon CdTe/CdS solar cell, Solar Energy Materials and Solar Cells 69 (2001) 61-68.

[11] B.C. Hong,, K. Kawano, PL and PLE studies of KMgF3:Sm crystal and the effect of its wavelength conversion on CdS/CdTe solar cell, Solar Energy and Materials and Solar Cells 80 (2003) 417-432.

[12] Hong, B.C., Kawano, K., Organic dye-doped thin films for wavelength conversion and their effects on the photovoltaic characteristics of CdS/CdTe solar cell, Japanese Journal of Applied Physics 43 (2004) 1421-1426.

[13] G.C. Glaeser, U. Rau, Improvement of photon collection in $\mathrm{Cu}(\mathrm{InGa}) \mathrm{Se}_{2}$ solar cells and modules by fluorescent frequency conversion, Thin Solid Films 515 (2007) 5964-5967.

[14] E. Klampaftis, D. Ross, S. Seyrling, A. Tiwari, B.S. Richards, Increase in short-wavelength response of encapsulated CIGS devices by doping the encapsulation layer with luminescent materials, Solar Energy Materials and Solar Cells 101 (2012) 62-67.

[15] E. Klampaftis, D. Ross, K.R. McIntosh, B.S. Richards, Enhancing the performance of solar cells via luminescent down-shifting of the incident spectrum: A review, Solar Energy Materials and Solar Cells 93 (2009) 1182-1194.

[16] B.S. Richards, K.R. McIntosh, Overcoming the poor short wavelength response of CdS/CdTe photovoltaic modules via luminescence down-shifting: ray tracing simulations, Progress in Photovoltaics: Research and Applications 15 (2006) 27-34. 
[17] J. S. Batchelder, A., H. Zewail, T. Cole, Luminescent solar concentrators. 1: theory of operation and techniques for performance evaluation, Journal of Applied Optics 20 (1979) 3733-3110.

[18] P. Kittidachachan, L. Danos, T.J.J. Meyer, N. Alderman, T. Markvart, Photon collection efficiency of fluorescent solar collectors, Chimia 61 (2007) 780-786.

[19] A. De Vos, A. Szymanska and V. Badescu, Modelling of solar cells with down-conversion of high energy photons, anti-reflection coatings and light trapping, Energy Conversion and Management 50 (2009) 328-336.

[20] V. Badescu, A. De Vos, A. M. Badescu and A. Szymanksa, Improved model for solar cells with down-conversion and down-shifting of high-energy photons, Journal of Physics D: Applied Physics 40 (2007) 341-352.

[21] V. Badescu and A. De Vos, Influence of some design parameters on the efficiency of solar cells with down-conversion and down shifting of high-energy photons, Journal of Applied Physics 102 (2007) 073102 1-4.

[22] W.H. Weber, J. Lambe, Luminescent greenhouse collector for solar radiation, Applied Optics 15 (1976) $2299-2300$.

[23] N.L. Boling, Luminescent solar collector structure, United States Patent 4190465 (1980).

[24] T. Oska, M. Hamalainen, P. Saarinen, J. Harkonen, A modified luminescent solar concentrator, Proceedings of SPIE, Optical Materials Technology for Energy Efficiency and Solar Energy Conversion V 15 April 1986 Innsbruck, Austria 114-118.

[25] K. Sakuta, S. Sawata, M. Tanimoto, Luminescent concentrator module of a practical size, $1^{\text {st }}$ World Conference on Photovoltaic Energy Conversion 5 - 9 December 1994 Hawaii, USA 1115-1118.

[26] A.F. Mansour, On enhancing the efficiency of solar cells and extending their performance life, Polymer Testing 22 (2003) 491-495.

[27] C. Corrado, S.W. Leow, M. Osborn, E. Chan, B. Balaban, S.A. Carter, Optimisation of gain and energy conversion efficiency using front-facing photovoltaic cell luminescent solar concentrator design, Solar Energy Materials and Solar Cells $111(2013) 74-81$.

[28] U. Rau, F. Einsele, G.C. Glaeser, Efficiency limits of photovoltaic fluorescent collectors, Applied Physics Letters 87 (2005) 171101.

[29] J.R. Mallinson, P.T. Landsberg, Meteorological effects on solar cells, Proceedings of the Royal Society London A 355 (1977) 115-130.

[30] G. Lifante, F. Cusso, F. Meseguer, F. Jaque, Solar concentrators using total internal reflection, Applied Optics 22 (1983) 3966-3970.

[31] A.V. Semichaevsky, H.T. Johnson, J. Yoon, R.G. Nuzzo, L. Li, J. Rogers, Theory for optimal design of waveguiding light concentrators in photovoltaic microcell array, Applied Optics 50 (2011) 2799-2808. 
[32] N. Muller, J. Manassen, Fluorescent window as wavelength shifter for a polysulfide containing photoelectrochemical cell, Solar Energy Materials 9 (1983) 207-216.

[33] D. Ross, D. Alsonson-Álvarez, E. Klampaftis, J. Fritsche, M. Bauer, M.G. Debije, R.M. Fitfield, B.S. Richards, The impact of luminescent down shifting on the performance of CdTe photovoltaics: impact of the module vintage, IEEE Journal of Photovoltaics 4 (2013) 457-464.

[34] T. Förster, Intermolecular energy migration and fluorescence, Annals of Physics 2 (1948) 55-75.

[35] P.D. Laible, R.S. Knox, T.G. Owens, Detailed balance in Förster-Dexter excitation transfer and its application to photosynthesis, Journal of Physical Chemistry 109 (1998) 1641-1648.

[36] L. Danos, T. Parel, T. Markvart, V. Barrioz, W.S.M. Brooks, S.J.C. Irvine, Increased efficiencies on CdTe solar cells via luminescent down-shifting with excitation energy transfer between dyes, Solar Energy Materials and Solar Cells 98 (2012) $486-490$.

[37] S.J.C. Irvine, V. Barrioz, D. Lamb, E.W. Jones, R.L. Rowlands-Jones, MOCVD of thin film photovoltaic solar cells-next-generation production technology? Journal of Crystal Growth 310 (2008) 5198-5203. 